\title{
A PROPORTIONALITY PRINCIPLE FOR PARTITIONING PROBLEMS
}

\author{
THEODORE P. HILL
}

(Communicated by William D. Sudderth)

\begin{abstract}
In a general class of measure-partitioning or fair-division problems, the extremal case occurs when the measures are proportional. Applications are given to classical and recent fair-division problems, and to statistical decision theory, mathematical physics, Banach space theory, and inequalities for continuous random variables.
\end{abstract}

1. Introduction. In many measure-partitioning inequalities the critical case, where equality is attained, often occurs when the measures are proportional; this happens for example in "fair-division" or "cake-cutting" inequalities of Neyman [8], Steinhaus, Banach and Knaster [10], Dubins and Spanier [2], and Hill [4, 5, 6]. The main purpose of this note is to prove the following proportionality principle for nonatomic measures (Theorem 1), and to give a number of applications of the theorem.

Of course, if the measures involved are all probability measures, proportionality means equality of the measures, and it is in this setting that the theorem will be stated. For arbitrary nonzero finite measures a simple rescaling of the measures yields the corresponding inequality; an example of this is seen in Corollary 4 below.

The following notation is used throughout this paper:

$\mu_{1}, \ldots, \mu_{n}$ are (countably additive, nonnegative) measures on a general measurable space $(S, \mathscr{F})$;

$\Pi_{k}=\Pi_{k}(\mathscr{F})$ is the collection of $\mathscr{F}$-measurable $k$-partitions $\left\{A_{i}\right\}_{i=1}^{k}$ of $S$;

$\mathscr{P}_{k}$ is the set of probabilities on $k$ points, i.e.,

$$
\mathscr{P}_{k}=\left\{\mathbf{p}=\left(p_{1}, \ldots, p_{k}\right) \in \mathbf{R}^{k}: p_{i} \geq 0 \forall i, \sum_{i=1}^{k} p_{i}=1\right\} ;
$$

$M_{n, k}$ is the set of real-valued $n \times k$ matrices; and

$\boldsymbol{\mu}(\hat{A})$ is the $n \times k$ matrix $\left(\mu_{i}\left(A_{j}\right)\right)_{i=1, \ldots, n ; j=1, \ldots, k}$, for $\hat{A}=\left\{A_{j}\right\}_{j=1}^{k} \in \Pi_{k}$.

In typical measure-partitioning or fair-division problems, a function $f: M_{n, k} \rightarrow \mathbf{R}$ is given and the best constant $C$ is sought so that

$$
\sup \left\{f(\boldsymbol{\mu}(\hat{A})): \hat{A} \in \Pi_{k}\right\} \geq C .
$$

Received by the editors February 6, 1987 and, in revised form, March 28, 1987.

1980 Mathematics Subject Classification (1985 Revision). Primary 60E15, 28B05; Secondary 90A05, 60A10.

Key words and phrases. Cake-cutting inequalities, fair-division problems, partitioning problems, proportionality principle, convexity theorem.

Research partially supported by NSF Grants DMS-86-01608 and 87-01691.

(C) 1988 American Mathematical Society $0002-9939 / 88 \$ 1.00+\$ .25$ per page 
If $C$ is known and the measures are nonatomic, the inequality (1) is usually easy to prove using the Convexity Theorem of Lyapounov [7] or a generalization of the convexity theorem (cf. Dvoretzky, Wald and Wolfowitz [3] or Dubins and Spanier [2]). The advantage of Theorem 1 below, which also is a consequence of Lyapounov's theorem, is that the best constant $C$ is identified as a "proportionality constant" depending only on $f$.

DEFINITION. For $f: M_{n, k} \rightarrow \mathbf{R}$, define

$$
C(f):=\sup \left\{f\left(\begin{array}{c}
\mathbf{p}_{1} \\
\vdots \\
\mathbf{p}_{n}
\end{array}\right): \mathbf{p}_{1}=\cdots=\mathbf{p}_{n}=\mathbf{p} \in \mathscr{P}_{k}\right\} .
$$

THEOREM 1. If $\mu_{1}, \ldots, \mu_{n}$ are nonatomic probability measures and $f$ is any real valued function on $M_{n, k}$, then

$$
\sup \left\{f(\boldsymbol{\mu}(\hat{A})): \hat{A} \in \Pi_{k}\right\} \geq C(f),
$$

and this bound is best possible. Moreover if $C(f)$ is attained for some $\mathbf{p}$ in $\mathscr{P}_{k}$, then there exists a measurable $k$-partition $\hat{A}$ of $S$ satisfying

$$
f(\boldsymbol{\mu}(\hat{A}))=C(f) .
$$

REMARKS. In many natural applications, such as those in the following section, $f$ can even be taken to be continuous, in which case the compactness of $\mathscr{P}_{k}$ implies that $C(f)$ is attained. For many problems, if the measures $\mu_{1}, \ldots, \mu_{n}$ are not proportional, the inequality in (2) can be shown to be strict (cf. Urbanik [12], Dubins and Spanier [2], and Hill [5]).

2. Applications of Theorem 1. Although it will not be explicitly stated every time, each of the inequalities (4)-(10) below is best possible, i.e., is attained for some $\left\{\mu_{i}\right\}$.

COROllary 1 (STEINHAUS, BANACH AND KNASTER [10]). If $\mu_{1}, \ldots, \mu_{n}$ are continuous probability measures, there is a measurable partition $\left\{A_{j}\right\}_{j=1}^{n}$ of $S$ satisfying

$$
\mu_{i}\left(A_{i}\right) \geq 1 / n \text { for all } i=1, \ldots, n .
$$

PROOF. Let $f: M_{n, n} \rightarrow \mathbf{R}$ be given by $f\left(\left(a_{i j}\right)\right)=\min _{i} a_{i i}$.

Then $C(f)=\sup \left\{\min _{i \leq n} p_{i}:\left(p_{1}, \ldots, p_{n}\right) \in \mathscr{P}_{n}\right\}=1 / n$, and since $f$ is continuous, (4) holds (even with equality) by (3).

(The elegant and practical solution in [10] is even constructive in the case of continuous measures, unlike the proof presented here which holds for the more general nonatomic case.)

COROLLARY 2 (NEYMAN [8]). If $\mu_{1}, \ldots, \mu_{n}$ are nonatomic probability measures, for each $k \geq 1$ there exists a measurable k-partition $\left\{A_{j}\right\}_{j=1}^{k}$ of $S$ satisfying

$$
\mu_{i}\left(A_{j}\right)=1 / k \text { for all } i=1, \ldots, n ; j=1, \ldots, k .
$$

PROOF. Let $f: M_{n, k} \rightarrow \mathbf{R}$ be given by

$$
f\left(\left(a_{i j}\right)\right)=-\max _{i, j, i^{\prime}, j^{\prime}}\left|a_{i j}-a_{i^{\prime} j^{\prime}}\right| .
$$


Then $C(f)=\sup \left\{-\max _{i, j}\left|p_{i}-p_{j}\right|:\left(p_{1}, \ldots, p_{k}\right) \in \mathscr{P}_{k}\right\}=0$, so since $f$ is continuous, (3) implies the existence of a partition $\left\{A_{j}\right\}_{j=1}^{k}=\hat{A} \in \Pi_{k}$ with $f(\boldsymbol{\mu}(\hat{A}))=0$, which implies that $\mu_{i}\left(A_{j}\right)=\mu_{1}\left(A_{1}\right)$ for all $i=1, \ldots, n$ and $j=1, \ldots, k$. Since the $\left\{\mu_{i}\right\}$ are probabilities, this establishes (5).

COROLlaRY 3 (DUBINS AND SPANIER [2]). If $\mu_{1}, \ldots, \mu_{n}$ are nonatomic probability measures, and $\alpha \in \mathscr{P}_{n}$, then there exists a measurable $n$-partition $\left\{A_{j}\right\}_{j=1}^{n}$ of $S$ satisfying

$$
\mu_{i}\left(A_{i}\right) \geq \alpha_{i} \text { for all } i=1, \ldots, n .
$$

PROOF. Let $f: M_{n, n} \rightarrow \mathbf{R}$ be given by

$$
f\left(\left(a_{i j}\right)\right)=\max _{j}\left|a_{j j}-\alpha_{j}\right| .
$$

It follows easily that $C(f)=0$, so (5) holds (even with equality) by (3), since $f$ is continuous.

The next result is a close analog of the "harmonic mean" theorem in Hill [5]; $\lambda$ is any nonatomic measure on $(S, \mathscr{F}) ;\|g\|_{p}$ is the $L_{p}$-norm $\left(\int|g|^{p} d \lambda\right)^{1 / p}$ of $g$ and $\|g\|=\|g\|_{1}$; and $I_{A}$ is the indicator function of $A$.

COROLlaRY 4. Let $f_{1}, \ldots, f_{n} \in L_{p}(\lambda), p>0$. Then there exists a measurable partition $\left\{A_{i}\right\}_{i=1}^{n}$ of $S$ satisfying

$$
\left\|f_{i} I_{A_{i}}\right\|_{p} \geq\left(\left\|f_{1}\right\|_{p}^{-p}+\cdots+\left\|f_{n}\right\|_{p}^{-p}\right)^{-1 / p} \quad \text { for all } i=1, \ldots, n .
$$

ProOF. Assume $p=1$; the proof for general $p>0$ is similar. If $\left\|f_{i}\right\|=0$ for some $i$, then $f_{i} \stackrel{\text { a.s. }}{=} 0$ and the result is trivial, so assume $m_{i}:=\left\|f_{i}\right\|>0$ for all $i=1, \ldots, n$. Let $\mu_{i}(\cdot)=m_{i}^{-1} \int_{(\cdot)}\left|f_{i}\right| d \lambda$, and observe that $\mu_{1}, \ldots, \mu_{n}$ are nonatomic probability measures on $(S, \mathscr{F})$. Define $f: M_{n, n} \rightarrow \mathbf{R}$ by

$$
f\left(\left(a_{i j}\right)\right)=\min _{i \leq n} m_{i} a_{i i}
$$

It follows easily [5, Lemma 2.2] that $C(f)=\left(m_{1}^{-1}+\cdots+m_{n}^{-1}\right)^{-1}$, so since $f$ is continuous (3) implies the existence of a measurable partition $\left\{A_{i}\right\}_{i=1}^{n}$ of $S$ satisfying

$$
\min _{i \leq n} m_{i} \mu_{i}\left(A_{i}\right)=\left(m_{1}^{-1}+\cdots+m_{n}^{-1}\right)^{-1} .
$$

Since $m_{i} \mu_{i}(A)=\int_{A}\left|f_{i}\right| d \lambda$, the conclusion (7) follows, even with equality.

(Note that for $p=1$, Corollary 4 is equivalent to Corollary 1.)

COROllaRY 5. Let $X_{1}, X_{2}, \ldots, X_{n}$ be independent continuous random variables on a probability space $(\Omega, \mathscr{F}, P)$, and let $k$ be any integer $1 \leq k \leq n$. Then there exists a Borel set $A \subset \mathbf{R}$ satisfying

$$
P\left(X_{i} \in A \text { for } i \leq k \text { and } X_{i} \notin A \text { for } i>k\right) \geq\left(\frac{k}{n}\right)^{k}\left(\frac{n-k}{n}\right)^{n-k} \text {. }
$$

ProOF. Define $\mu_{1}, \ldots, \mu_{n}$ by $\mu_{i}(\cdot)=P\left(X_{i} \in(\cdot)\right)$, and define $f: M_{n, 2} \rightarrow \mathbf{R}$ by

$$
f\left(\left(a_{i j}\right)\right)=\prod_{i=1}^{k} a_{i 1} \prod_{i=k+1}^{n} a_{i 2} \text {. }
$$


It is easy to show that $C(f)=k^{k}(n-k)^{n-k} n^{-n}$, so (8) (with equality) follows from (3).

The next result is a problem from mathematical physics involving the equilibrium positions of $n+1$ unit "masses" in $[0,1]$ (see [11, p. 140]); here $\mu_{i}\left(A_{i}\right)$ represents the "distance" between the $i$ th mass and the $(i-1)$ st mass.

THEOREM 2. If $\mu_{1}, \ldots, \mu_{n}$ are nonatomic probability measures, then there exists a measurable n-partition $\left\{A_{i}\right\}_{i=1}^{n}$ of $S$ satisfying

$$
\prod_{\substack{i, j=1, \ldots, n \\ i<j}}\left(\mu_{i}\left(A_{i}\right)+\cdots+\mu_{j}\left(A_{j}\right)\right) \geq \prod_{\substack{i, j=1, \ldots, n \\ i<j}}\left|C_{j}-C_{i}\right|
$$

where $C_{1}, \ldots, C_{n}$ are the zeroes of the (rescaled) Jacobi polynomial $P_{n}(2 x-1)$.

Proof. Define $f: M_{n, n} \rightarrow \mathbf{R}$ by

$$
f\left(\left(a_{i j}\right)\right)=\prod_{\substack{i, j=1, \ldots, n \\ i<j}}\left(a_{i i}+\cdots+a_{j j}\right)
$$

By Theorem 6.7 .1 of $[\mathbf{1 1}], C(f)$ is the constant on the right-hand side of (9); the conclusion follows as before from (3).

The final application of Theorem 1 is to the classification problem of statistical decision theory. A random variable $X$ has one of the known distributions $\mu_{1}, \ldots, \mu_{n}$, but it is not known which. A single observation (realization) $X(\omega)$ is made, and it then must be guessed from which of the $n$ distributions the observation came. A decision rule corresponds to a measurable partition $\left\{A_{i}\right\}_{i=1}^{n}$ of $\mathbf{R}$ ("if $X(\omega) \in A_{i}$, guess distribution $\left.\mu_{i}{ }^{\prime \prime}\right)$, and a loss $L(i, j)$ is incurred if the guess is $\mu_{j}$ and the true distribution is $\mu_{i}$. The objective is to minimize the maximum expected loss

$$
R(L ; \boldsymbol{\mu})=\inf \left\{\max _{i \leq n} \sum_{j=1}^{n} L(i, j) \mu_{i}\left(A_{j}\right):\left\{A_{i}\right\}_{i=1}^{n} \in \Pi_{n}(\mathbf{R}, \mathbf{B})\right\} .
$$

COROLLARY 6. Let $\mu_{1}, \ldots, \mu_{n}$ be continuous probability distributions, and let $L \in M_{n, n}$. Then

$$
R(L ; \boldsymbol{\mu}) \leq \inf \left\{R(L, \mathbf{p}): \mathbf{p} \in \mathscr{P}_{n}\right\}=V(L),
$$

where $R(L, \mathbf{p})$ is $\max _{i \leq n}\left\{\sum_{j=1}^{n} L(i, j) p_{j}\right\}$, the maximum loss of one player in a game using mixed strategy $\mathbf{p}$ against strategies (pure or mixed) of the opponent, and $V(L)$ is the usual value of the game in mixed strategies when the payoff matrix is $-L$. Moreover this bound is best possible.

Proof. Define $f: M_{n, n} \rightarrow \mathbf{R}$ by

$$
f\left(\left(a_{i j}\right)\right)=-\max _{i \leq n} \sum_{j=1}^{n} L(i, j) a_{i j}
$$

and apply Theorem 1 . 
3. Proof of Theorem 1. Fix $f: M_{n, k} \rightarrow \mathbf{R}$, and $\mathbf{p}=\left(p_{1}, \ldots, p_{k}\right) \in \mathscr{P}_{k}$. Since the $\left\{\mu_{i}\right\}$ are nonatomic, Lyapounov's Theorem implies that the range $R(\boldsymbol{\mu})=$ $\left\{\left(\mu_{1}(A), \ldots, \mu_{n}(A)\right): A \in \mathscr{F}\right\}$ is a convex (and compact) subset of $\mathbf{R}^{n}$. Taking $A=\varnothing$ and $A=S$ shows that $(0,0, \ldots, 0)$ and $(1,1, \ldots, 1)$ are in $R(\boldsymbol{\mu})$, so (by convexity) $\left(p_{1}, p_{1}, \ldots, p_{1}\right) \in \mathbf{R}(\boldsymbol{\mu})$, which implies that there is a set $A_{1} \in \mathscr{F}$ with $\mu_{1}\left(A_{1}\right)=\cdots=\mu_{n}\left(A_{1}\right)=p_{1}$. Apply this same argument next to $S \backslash A_{1}$ to obtain a set $A_{2} \in \mathscr{F}$, disjoint from $A_{1}$, which satisfies $\mu_{1}\left(A_{2}\right)=\cdots=\mu_{n}\left(A_{2}\right)=p_{2}$, and then to $S \backslash\left(A_{1} \cup A_{2}\right)$, etc. to obtain a $k$-partition $\hat{A}=\left\{A_{j}\right\}_{j=1}^{k} \in \mathscr{F}$ satisfying

$$
\boldsymbol{\mu}(\hat{A})=\left(\begin{array}{c}
\mathbf{p} \\
\vdots \\
\mathbf{p}
\end{array}\right) .
$$
(3).

Since $\mathbf{p} \in \mathscr{P}_{k}$ was arbitrary, applying $f$ to $\boldsymbol{\mu}(\hat{A})$ completes the proof of $(2)$ and

(An alternate short proof of Theorem 1 can also be based on the "matrixconvexity" results in [2 or 3]; the proof given above is more elementary in that it depends only on the classical convexity theorem.)

Although in some partitioning problems with atoms the extremal case is also the proportional one (e.g., Hill [6]), in general the conclusion of Theorem 1 may fail if the measures have atoms, even if $f$ is continuous.

EXAMPLE 1. Let $S=\{a, b\}, \mathscr{F}=\{\varnothing,\{a\},\{b\}, S\} ;$ let $\mu_{1}(\{a\})=\mu_{2}(\{b\})=1$, $\mu_{1}(\{b\})=\mu_{2}(\{a\})=0$; and let $f: M_{2,2} \rightarrow \mathbf{R}$ be given by

$$
f\left(\begin{array}{ll}
a_{1} & a_{2} \\
a_{3} & a_{4}
\end{array}\right)=\min _{i}\left(1-a_{i}\right) .
$$

Then

$$
\sup \left\{f(\mu(\hat{A})): \hat{A} \in \Pi_{2}\right\}=0<1 / 2=f\left(\begin{array}{cc}
1 / 2 & 1 / 2 \\
1 / 2 & 1 / 2
\end{array}\right)=C(f) .
$$

REMARK. All of the main results of this paper remain valid in the setting of nonatomic finitely additive measures on $\sigma$-algebras or the somewhat more general class of algebras considered by Armstrong and Prikry [1], who showed that the convexity conclusion of Lyapounov's theorem holds in that more general setting. In Corollary 4 , if $\lambda$ is only finitely additive, $V_{p}$ functions may be more appropriate than $L_{p}$ functions (see Chapter 7 of [9]), especially if $\lambda$ is finite.

ACKNOWLEDGMENT. The author is grateful to Professor J. Geronimo for several conversations concerning Theorem 2 , and to the referee for several suggestions and corrections.

\section{REFERENCES}

1. T. Armstrong and K. Prikry, Liapounoff's Theorem for nonatomic, finitely-additive, bounded finite-dimensional vector-valued measures, Trans. Amer. Math. Soc. 266 (1981), 499-514.

2. L. Dubins and E. Spanier, How to cut a cake fairly, Amer. Math. Monthly 68 (1961), 1-17.

3. A. Dvoretzky, A. Wald and J. Wolfowitz, Relations among certain ranges of vector measures, Pacific J. Math. 1 (1951), 59-74.

4. T. Hill, Determining a fair border, Amer. Math. Monthly 90 (1983), 438-442.

5. __ Equipartitioning common domains of nonatomic measures, Math. Z. 189 (1985), 415-419. 
6. __ Partitioning general probability measures, Ann. Probab. 15 (1987), 804-813.

7. A. Lyapounov, Sur les fonctions-vecteurs complètement additives, Bull. Acad. Sci. URSS 4 (1940), 465-478.

8. J. Neyman, Un théorème d'existence, C. R. Acad. Sci. Paris Ser. A-B 222 (1946), 843-845.

9. K. Rao and M. Rao, Theory of charges, Academic Press, New York, 1983.

10. H. Steinhaus, Sur la division pragmatique, Econometrica (Supplement) 17 (1949), 315-319.

11. G. Szegö, Orthogonal polynomials, Amer. Math. Soc. Colloq. Math. Publ., vol. 23, Amer. Math. Soc., Providence, R. I., 1978.

12. K. Urbanik, Quelques théorèmes sur les mesures, Fund. Math. 41 (1955), 150-162.

School of Mathematics, Georgia institute of Technology, Atlanta, GeorGIA 30332 